Neurosurg Focus 17 (6):E4, 2004

\title{
Surgical strategies for vertebral osteomyelitis and epidural abscess
}

\author{
Patrick C. Hsieh, M.D., Robert J. Wienecke, M.D., Brian A. O’Shaughnessy, M.D., \\ Tyler R. Koski, M.D., AND STePhen L. ONDRA, M.D. \\ Department of Neurological Surgery, The Feinberg School of Medicine and McGaw Medical Center, \\ Northwestern University, Chicago, Illinois
}

\begin{abstract}
Pyogenic vertebral discitis and osteomyelitis (PVDO) has become an increasing problem for the spine surgeon. Despite recent advances in medical care and improved diagnostic neuroimaging, PVDO remains a major cause of illness and death in the elderly population. Infection of the spinal column often presents insidiously; however, if not treated appropriately and in a timely manner it can lead to severe neurological impairment, systemic septicemia, and progressive spinal deformity. In this paper the authors review the epidemiological and pathophysiological features and the clinical presentation of PVDO. Conventional medical therapy is described, with a particular focus on the methods of diagnosis. Surgical strategies for PVDO are then presented based on the literature and according to the practice of the senior author (S.L.O.), with an emphasis placed on structural considerations, implant selection, and techniques for augmenting vascular tissue to the site of infection.
\end{abstract}

\section{KEY WORDS • epidural abscess • kyphotic deformity • spinal deformity • vertebral osteomyelitis}

Historically, PVDO has been considered to be an uncommon clinical entity. In one large study, ${ }^{2}$ the incidence of this disease was found to be 2.2 per 100,000 population per year. Nevertheless, PVDO is thought to represent at least 2 to $8 \%$ of all cases of osteomyelitis. ${ }^{26}$ The demographics of the patient population affected by the disease have changed over the years, and the incidence of PVDO appears to be steadily increasing. Several elements of current medical practice are likely to account for this epidemiological change, including improved neuroimaging, increased awareness of the condition, and greater longevity of the general population. The widespread use of chemotherapy for cancer and immunotherapy for the human immunodeficiency virus also may play a role. In addition, the common use of invasive spinal procedures, both as diagnostic and therapeutic techniques, are important factors responsible for the increased incidence and the change in patient demographics compared with the early 20 th century. ${ }^{4,25,26}$

Risk factors associated with the development of PVDO include advanced age, male sex, immunosuppression, and intravenous drug abuse. In addition, patients with significant medical comorbidities such as diabetes mellitus are clearly prone to spinal infection. The peak incidence of PVDO occurs in the sixth and seventh decades and is relatively rare in the young population. The majority of pa-

Abbreviations used in this paper: $\mathrm{CT}=$ computerized tomography; $\mathrm{MR}=$ magnetic resonance; $\mathrm{PVDO}=$ pyogenic vertebral discitis and osteomyelitis; $\mathrm{VB}=$ vertebral body. tients afflicted early in life have a history of immunosuppression or intravenous drug abuse. The incidence is higher in men, with a male/female ratio of $2: 1 .^{24,25}$ As stated, patients with chronic systemic diseases and immunocompromised states are at increased risk for the development of PVDO; such conditions include diabetes mellitus, corticosteroid drug use, chemotherapy, rheumatological or immunological diseases, hepatic or renal failure, malnutrition, and myelodysplastic disease. . $^{410,19,24,26}$

\section{Pathophysiological Mechanisms}

Hematogenous dissemination secondary to bacteremia appears to be an extremely common pathophysiological mechanism leading to vertebral osteomyelitis. ${ }^{26,30}$ Bloodborne pathogens may travel to the spinal column, either through the arterial or venous system. The genitourinary tract is often the most common identifiable source of the infection, particularly in elderly patients. Other sources of infection include the upper respiratory tract, oral cavity, cutaneous ulcers, traumatic wounds, and surgical or procedure sites. ${ }^{4,10,24}$ Partly because of the comparatively larger VBs and disc spaces as well as their relative patterns of blood supply, the majority of PVDO cases involve the lumbar and thoracic spine. ${ }^{26}$ The lumbar spine is affected in approximately $57 \%$ of cases, whereas the thoracic spine is involved in $35 \% .{ }^{24,27}$

Although advancements in the surgical treatment of vertebral osteomyelitis were initially driven by an attempt to improve the management of tuberculous spinal osteomyelitis (Pott disease), the vast majority of PVDO is actu- 
ally caused by Gram-positive organisms, including species of Staphylococcus and Streptococcus. Staphylococcus aureus accounts for one half to two thirds of the cases in most clinical series previously reported., ${ }^{4,24}$ Nevertheless, Gram-negative and atypical organisms, including Escherichia coli, Haemophilus influenzae, Proteus species, enterococci, and various fungi are now frequently isolated. ${ }^{6,8,23,28}$ Pseudomonas species are the most common causative organisms for PVDO in intravenous drug abusers. ${ }^{4,20,26}$

Vertebral osteomyelitis typically begins with hematogenous metastasis of microbial organisms to the richly vascularized vertebral metaphysis. ${ }^{30} \mathrm{~A}$ second possible route of infection is through the paraspinous venous plexus described by Batson. ${ }^{1,18}$ Once infection is established, it may spread to the intervertebral disc, surrounding myofascial tissues, and epidural space. With progression, several additional sites of infection may emerge, including epidural abscess, subdural abscess, paraspinal abscess, and meningitis. Moreover, destruction of the VB and intervertebral disc may lead to spinal deformity and instability, factors that clearly place the spinal cord and surrounding neural and vascular structures at risk. Iatrogenic kyphotic deformity is particularly common in the thoracic spine when patients with infection and weakening of the anterior spinal column are treated surgically with a procedure involving posterior tension band destabilization (that is, multilevel laminectomies). These patients are highly susceptible to neurological deterioration because of the ky- photic deformity and as a rule require circumferential spinal stabilization (Fig. 1).

\section{CLINICAL PRESENTATION AND DIAGNOSIS}

The clinical presentation of vertebral osteomyelitis can be nonspecific, but its cardinal clinical feature is local pain and muscle spasm, which is present in more than $90 \%$ of patients. ${ }^{24-26}$ Far less common symptoms include chest, abdominal, hip, or radicular pain that can at times overshadow the back pain and lead to a delay in diagnosis. ${ }^{10,25}$ Neurological deficit associated with PVDO occurs in 6 to $17 \%$ of cases. ${ }^{4,7,10,26}$ Factors that predispose patients to neurological compromise include $S$. aureus infection, diabetes mellitus, rheumatoid arthritis, systemic steroid therapy, and age older than 50 years., ${ }^{4,26}$ Neurological deficits can result from direct compression of the spinal cord secondary to spinal instability, septic thrombosis of spinal vascular plexus, or inflammatory infiltration.

Diagnosis of PVDO is often delayed, even in the modern era of advanced medical care and sophisticated neuroimaging. Fever and leukocytosis are found in only 40 to $50 \%$ of patients. An elevated erythrocyte sedimentation rate is considered to be the most common laboratory abnormality; $5,21,26$ however, we have found that the C-reactive protein level is a more valuable serum marker to follow during therapy because of its temporal pattern in the blood stream and comparatively quicker normalization with effective treatment. ${ }^{21}$ Neuroimaging studies obtained
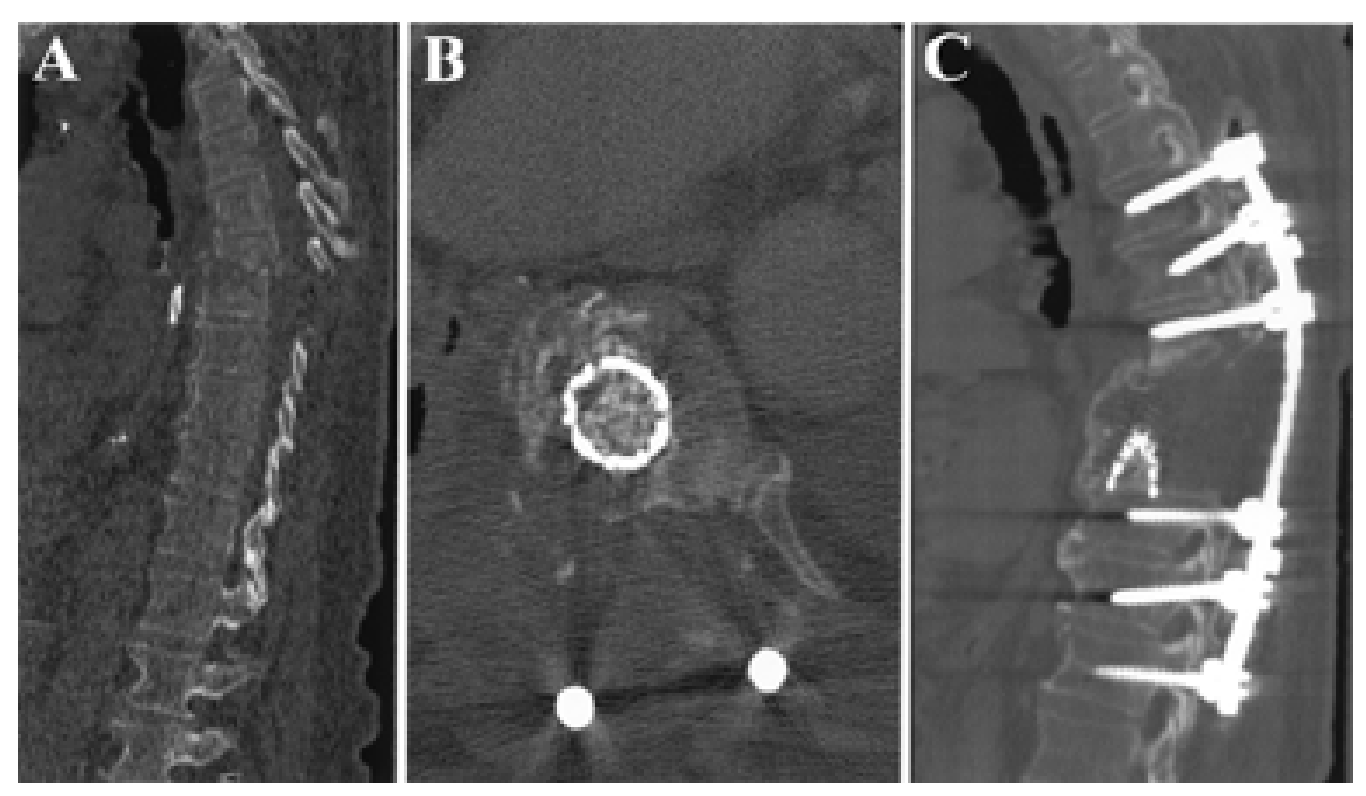

Fig. 1. Neuroimages obtained in a 71-year-old man with multiple medical comorbidities (including poorly controlled diabetes mellitus) who presented to another institution with symptoms that included several days of fever, midline thoracic pain, and progressive leg weakness. A diagnosis of thoracic osteomyelitis with epidural abscess was made and the patient was treated with thoracic laminectomies. Unfortunately, he failed to improve and his leg weakness worsened to near paraplegia over the ensuing weeks. A: Admission CT scan with sagittal reconstruction obtained when the patient presented to our institution, demonstrating kyphotic deformity at the site of the laminectomies. B and C: The patient was treated surgically with anterior corpectomy, placement of a titanium mesh cage filled with autograft (B), and multilevel segmental instrumentation posteriorly (C). 
in the early course of the disease do not demonstrate the characteristic finding of disc space narrowing, local osteopenia, lytic VB destruction, and/or spinal deformity. Neuroimaging findings typically lag behind the clinical course by 2 to 3 months. Findings on CT scanning have confirmed that this is more sensitive than other imaging modalities for detecting bone changes and can clearly demonstrate paraspinal soft-tissue swelling or abscess. Nevertheless, MR imaging has been shown to have the highest sensitivity, specificity, and accuracy in the diagnosis of vertebral osteomyelitis. ${ }^{17}$ Other imaging techniques available include ${ }^{99 \mathrm{~m} T c}$ bone scans, ${ }^{67} \mathrm{Ga}$ - or ${ }^{111} \mathrm{In}$-labeled leukocyte scans, or single-photon emission computerized tomography scans. ${ }^{29}$

The single most important study for the diagnosis of PVDO is tissue sampling. ${ }^{26}$ Diagnosis, however, can be firmly established without direct biopsy sampling in patients with positive blood cultures, but bacterial growth on blood cultures occurs in less than $25 \%$ of cases. ${ }^{14,24,25}$ Percutaneous needle biopsy sampling of the infected VB can be performed under fluoroscopic or CT guidance and is diagnostic in 68 to $86 \%$ of cases. ${ }^{10,12,25}$ In cases in which percutaneous biopsy sampling fails, is nondiagnostic, or cannot be performed, an open surgical biopsy procedure can be performed. This allows the surgeon to obtain a large pathological specimen under direct visualization, which may lead to more success in isolation of an organism.

\section{Conventional Medical Management}

Broad-spectrum antibiotic drugs should be administered after materials for culture are obtained. Blood and image-guided needle aspirates of the infected space should be sent for culture to identify the microorganism and its sensitivities. Also, a baseline C-reactive protein level and the erythrocyte sedimentation rate may be obtained to guide ongoing antimicrobial therapy. Antibiotic coverage is tailored to treat the organism type and its drug sensitivity. Patients with spinal infections have increased metabolic needs and so should receive adequate nutrition.

\section{SURGICAL TREATMENT}

\section{General Principles}

Surgical indications include symptomatic compression of neural structures, structural instability, spinal deformity, and medically intractable pain. Discitis without structurally significant osteomyelitis that does not threaten nervous tissue may be treated with antibiotic medications alone. In general, cervical and thoracic epidural abscess should be treated by surgical decompression. Lumbar epidural abscess may be treated medically if there is no evidence for cauda equina or conus dysfunction. Vertebral osteomyelitis associated with structural instability typically requires surgery. If neurological instability exists, decompression and instrumented spinal stabilization may be performed in the same procedure, even in the setting of acute pyogenic infection. ${ }^{3,21}$ If the patient is neurologically stable but structural instability is anticipated, instrumentation may be performed on an elective basis at a later date. If deformity and pain are present in a patient without neurological compromise, correction of deformity and placement of instrumentation may be delayed until the infection has been cleared with antimicrobial drugs. Of course, many instances exist in which surgical debridement is required for clearance of infection.

\section{Structural Considerations in PVDO}

In the face of pyogenic vertebral element destruction, anterior spinal column reconstruction is required. Avoidance of structural allograft is recommended because it may serve as a nidus for ongoing infection. Structural autograft or morselized autograft packed into a titanium cage is preferred for this reason. If the posterior tension band is intact, anterior column restructuring alone may be adequate; however, if the posterior tension band has been destroyed, either iatrogenically or by infection, posterior instrumentation should be implemented (Fig. 2).

\section{Titanium Compared With Stainless Steel Implants}

The metal selection for the implant should also be considered. In infected areas, titanium and titanium alloys are superior to stainless steel based on their imaging characteristics and ability to allow for soft-tissue ingrowth. Titanium and titanium alloy produce less artifact on MR imaging, which is the modality most commonly used in follow-up review of spinal infections. Long-term clearance of infection is more likely with the use of titanium or titanium alloy implants than with stainless steel implants, because the porous nature of titanium may allow for and facilitate soft-tissue attachment and the delivery of adequate concentrations of antimicrobial drugs. With stainless steel implants, a pseudocapsule often forms that can harbor bacteria.

\section{Wound Drainage}

We close all surgical wounds over "clot-stop" drains (Axiom Medical, Inc., Rancho Dominguez, CA). For posterior wounds we place two submuscular drains, and if there has been significant separation of the skin and underlying fascia, subcutaneous drains are placed. For infected wounds or surgical wounds created during the treatment of the spinal infection, we work with the Plastic Surgery Service to achieve optimal closure. The goal is to achieve closure with adequate soft-tissue transfer into the wound and no wound closure tension. Pedicled flaps are used for both dead-space obliteration and as a method to improve blood supply to the devitalized remaining soft tissue when needed.

\section{Delivery of Vascularized Tissue to the Infection}

In our experience, delivery of vascularized tissue to the infected area is the key to long-term success in a patient affected by vertebral osteomyelitis. A corollary of this premise is to ensure a two-layer closure. ${ }^{9}$ We attempt to place a well-vascularized layer of skin and muscle over the spine and implants. Each spinal region provides separate challenges and opportunities to the realization of these goals. Vascularized grafts may be described as structural or nonstructural, and rotational or free. It is sometimes possible, if not preferable, to provide both vascularized structural and nonstructural grafts to an area of infection. In several challenging cases of spinal osteomyelitis involving the thoracic spine, we have used a pedicled rib flap placed inside a titanium mesh cage as the 


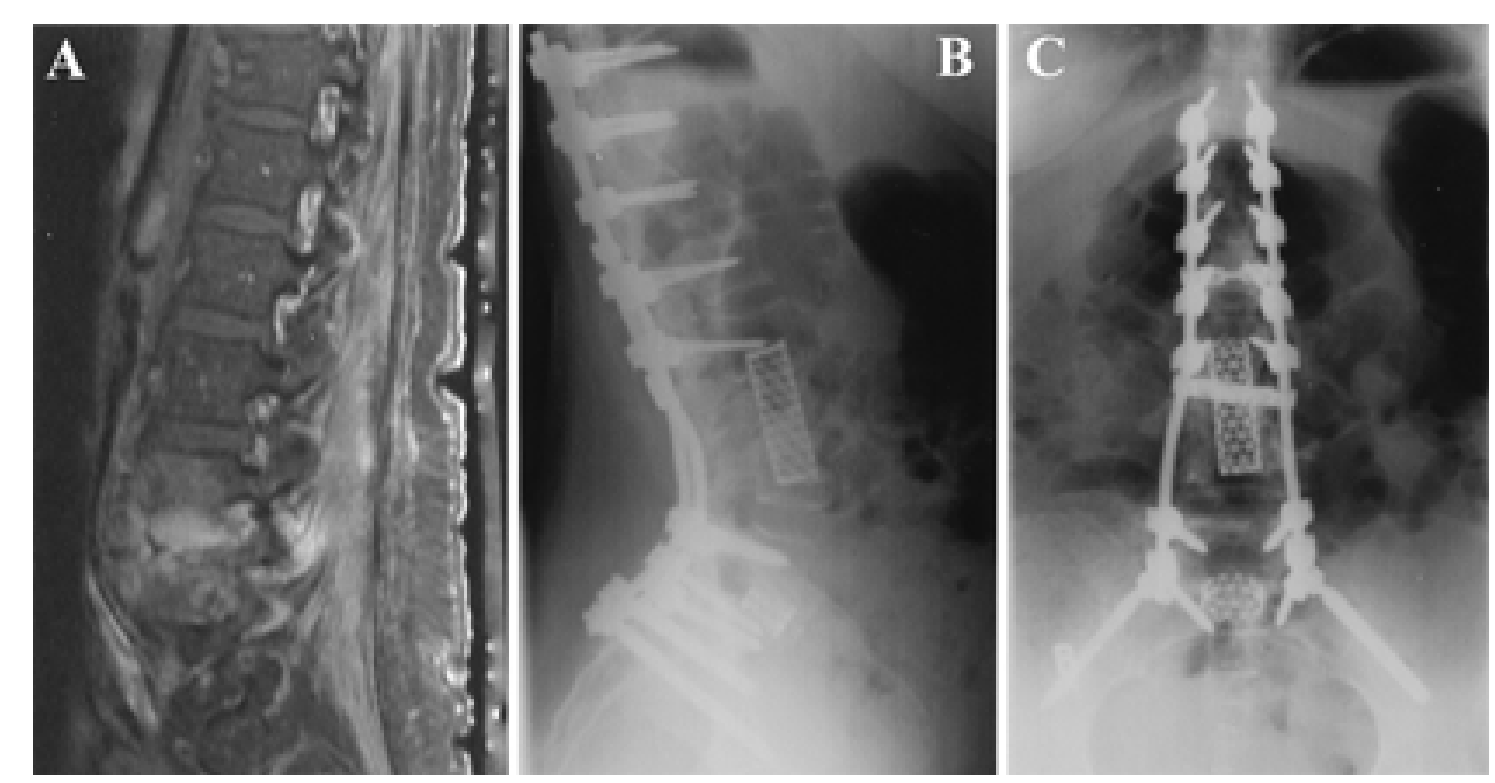

Fig. 2. Neuroimages obtained in a 57-year-old woman with several medical comorbidities in whom severe, destructive spinal osteomyelitis associated with profound lumbosacral instability developed, as demonstrated on preoperative dynamic imaging. A: Preoperative MR image revealing the severity of the destructive osteomyelitic process. B and C: Postoperative lateral (B) and anteroposterior (C) x-ray films obtained after completion of circumferential spinal stabilization including bilateral iliac fixation.

interbody construct. This graft offers the benefit of the structural support provided by the cage, coupled with maximum fusion potential offered by the vascularized bone flap. In addition, the vascularized rib graft theoretically enables better antibiotic delivery to the region of spinal infection because of the additional blood flow pro- vided. Again, vascularized tissue provides an ideal environment for clearance of bone infection and ultimately healing.

Vascularized Structural Grafts. Vascularized structural autograft offers the advantages of resistance to infection and rapid bone incorporation. In the cervical spine, the re-

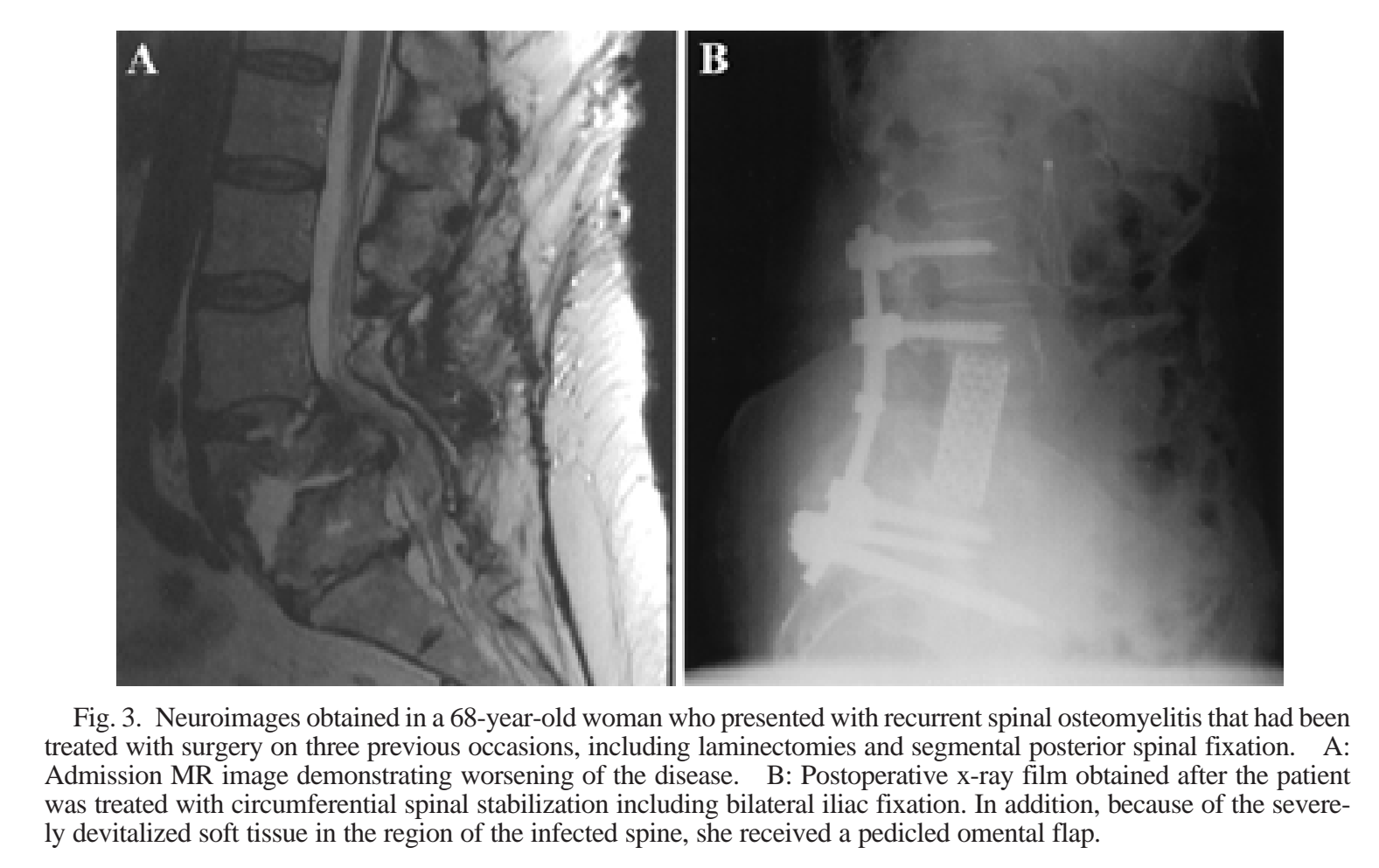


gional anatomy is such that grafts are typically of the free type. We have successfully used fibular vascularized autograft in the cervical spine. Structural autograft in the thoracic spine may be rotational or free. As described, rotational rib graft within a titanium cage has been used successfully at our institution. As in the cervical spine, free fibular grafts may be used to restructure the thoracic spine.

Vascularized Nonstructural Grafts. Rotational trapezius or latissimus muscle flaps may be used for soft-tissue coverage. As described earlier, the erector spinae muscles may also be mobilized and used for complex wound closures. ${ }^{9}$ We have also used omentum flaps to bring vascularized tissue to the anterior or posterior lumbar spine and sacrum. As mentioned, at our institution the omentum is the most commonly used vascularized nonstructural graft for the treatment of spinal infection in the anterior column (Fig. 3). Because of its biochemical attributes, profound vascularity, bulk, and adherence it lends itself well to grafting. ${ }^{13,15,16}$ Distance from the abdomen requires omental flaps in the cervical spine to be free. We have used omentum to serve as a vascular barrier between the spine and the perforated esophagus. Rotational pectoralis muscle flaps may also be used in the anterior cervical spine. Nevertheless, in our experience omental flaps are associated with shorter time to closure, duration of stay, and return to oral feeding. ${ }^{22}$

We have also described the connection of a trapezius muscle flap posterior to the anterior cervical vasculature through a free cephalic vein arteriovenous conduit. ${ }^{11} \mathrm{La}-$ tissimus and trapezius muscle rotational flaps may also be used in the posterior cervical spine. If significant scarring and induration exists, the paraspinous muscles may need to be mobilized. Dumanian and colleagues ${ }^{9}$ describe the mobilization of the paraspinal muscles to allow their optimal use. Laterally, the fascia is incised, taking care to preserve the skin's vasculature. Medially, the muscle is undermined to allow its tension-free mobilization to the midline.

Lumbar osteomyelitic anterior column destruction may be addressed via a retroperitoneal approach. A titanium cage packed with autograft provides excellent anterior column support. Omentum pulled through a peritoneal window provides for vascularization at the lumbosacral junction. Gluteal and paraspinous muscle flaps may be used for posterior wound coverage. ${ }^{9}$

\section{CONCLUSIONS}

The treatment of vertebral osteomyelitis and epidural abscess represents one of the greatest challenges to the spine surgeon. Each case must be planned on an individual basis with the nuances of the particular patient kept in mind. Basic neurosurgical, orthopedic, and plastic surgery principles need to be meshed into a workable plan. The strategy must provide for neural decompression, spinal stabilization, and clearance of infection. The fear of using spinal instrumentation in grossly infected spaces is understandable, but in our experience and that of others, surgical success and clearance of infection are attainable. With modern surgical techniques the morbidity and mortality rates associated with severe spinal infection may be decreased. In addition to neural decompression and spinal stabilization, providing vascularized graft tissue to the infected area is integral to effective therapy.

\section{References}

1. Batson OV: The function of the vertebral veins and their role in the spread of metastases. Ann Surg 112:138-149, 1940

2. Beronius M, Bergman B, Andersson R: Vertebral osteomyelitis in Goteborg, Sweden: a retrospective study of patients during 1990-95. Scand J Infect Dis 33:527-532, 2001

3. Carragee EJ: Instrumentation of the infected and unstable spine: a review of 17 cases from the thoracic and lumbar spine with pyogenic infections. J Spinal Disord 10:317-324, 1997

4. Carragee EJ: Pyogenic vertebral osteomyelitis. J Bone Joint Surg Am 79:874-880, 1997

5. Carragee EJ, Kim D, van der Vlugt T, et al: The clinical use of erythrocyte sedimentation rate in pyogenic vertebral osteomyelitis. Spine 22:2089-2093, 1997

6. Collert S: Osteomylelitis of the spine. Acta Orthop Scand 48: 283-290, 1977

7. Colmenero JD, Jimenez-Mejias ME, Reguera JM, et al: Tuberculous vertebral osteomyelitis in the new millennium: still a diagnostic and therapeutic challenge. Eur J Clin Microbiol Infect Dis 23:477-483, 2004

8. Cone LA, Byrd RG, Potts BE, et al: Diagnosis and treatment of Candida vertebral osteomyelitis: clinical experience with a short course therapy of amphotericin B lipid complex. Surg Neurol 62:234-237, 2004

9. Dumanian GA, Ondra SL, Liu J, et al: Muscle flap salvage of spine wounds with soft tissue defects or infection. Spine 28: 1203-1211, 2003

10. Eismont FJ, Bohlman HH, Soni PL, et al: Pyogenic and fungal vertebral osteomyelitis with paralysis. J Bone Joint Surg Am 65:19-29, 1983

11. Few JW, Marcus JR, Lee MJ, et al: Treatment of hostile midline back wounds: an extreme approach. Plast Reconstr Surg 105:2448-2451, 2000

12. Garcia A Jr, Grantham SA: Hematogenous pyogenic vertebral osteomyelitis. Am J Orthop 42:429-436, 1960

13. Giordano PA, Griffet J, Argenson C: Pedicled greater omentum transferred to the spine in a case of postoperative infection. Plast Reconstr Surg 93:1508-1511, 1994

14. King DM, Mayo KM: Infective lesions of the vertebral column. Clin Orthop 96:248-253, 1973

15. Lantieri LA, Tantaoui B, Rimareix FA, et al: Lower back coverage with endoscopically harvested pedicled greater omental flap. Plast Reconstr Surg 103:960-963, 1999

16. MacMillan M, Stauffer ES: The effect of omental pedicle graft transfer on spinal microcirculation and laminectomy membrane formation. Spine 16:176-180, 1991

17. Modic MT, Feiglin DH, Piraino DW, et al: Vertebral osteomyelitis: assessment using MR. Radiology 157:157-166, 1985

18. Onuigbo WI: Batson's theory of vertebral venous metastasis: a review. Oncology 32:145-150, 1975

19. Osenbach RK, Hitchon PW, Menezes AH: Diagnosis and management of pyogenic vertebral osteomyelitis in adults. Surg Neurol 33:266-275, 1990

20. Patzakis MJ, Rao S, Wilkins J, et al: Analysis of 61 cases of vertebral osteomyelitis. Clin Orthop 264:178-183, 1991

21. Przybylski GJ, Sharan AD: Single-stage autogenous bone grafting and internal fixation in the surgical management of pyogenic discitis and vertebral osteomyelitis. J Neurosurg (Spine) 94:1-7, 2001

22. Reid RR, Dutra J, Conley DB, et al: Improved repair of cervical esophageal fistula complicating anterior spinal fusion: free omental flap compared with pectoralis major flap. Report of four cases. J Neurosurg (Spine) 100:66-70, 2004

23. Santos AB, Llamas P, Gadea I, et al: Aspergillus fumigatus: 
a rare cause of vertebral osteomyelitis. Haematologica 89: ECR10, 2004

24. Sapico FL, Montgomerie JZ: Pyogenic vertebral osteomyelitis: report of nine cases and review of the literature. Rev Infect Dis 1:754-776, 1979

25. Sapico FL, Montgomerie JZ: Vertebral osteomyelitis. Infect Dis Clin North Am 4:539-550, 1990

26. Slucky AV, Eismont FJ: Spinal infection, in Bridwell $\mathrm{KH}$, DeWald RL (eds): The Textbook of Spinal Surgery, ed 2. Philadelphia: Lippincott-Raven, 1997, Vol 2, pp 2141-2183

27. Spies EH, Stucker R, Reichelt A: Conservative management of pyogenic osteomyelitis of the occipitocervical junction. Spine 24:818-822, 1999

28. Tarr PE, Sakoulas G, Ganesan A, et al: Hematogenous enterococcal vertebral osteomyelitis: report of 2 cases and review of the literature. J Infect 48:354-362, 2004
29. Turpin S, Lambert R: Role of scintigraphy in musculoskeletal and spinal infections. Radiol Clin North Am 39:169-189, 2001

30. Wiley AM, Trueta J: The vascular anatomy of the spine and its relationship to pyogenic vertebral osteomyelitis. J Bone Joint Surg Br 41:796-809, 1959

Manuscript received October 28, 2004.

Accepted in final form November 4, 2004.

Address reprint requests to: Stephen L. Ondra, M.D., Department of Neurological Surgery, The Feinberg School of Medicine, Northwestern University, 676 St. Clair Street, 2210, Chicago, Illinois 60611. email: sondra@nmff.org. 